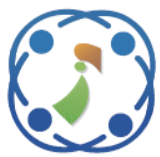

\title{
Resource Allocation Based on Matchmaking Services in Multiple Clouds Using Trustworthy and Scalable Service Providers Algorithm
}

\author{
Palaiyah Solainayagi ${ }^{1 *}$ \\ Ramalingam Ponnusamy ${ }^{2}$ \\ ${ }^{I}$ Sathyabama Institute of Science and Technology, Chennai, Tamilnadu, India, \\ ${ }^{2}$ CVR College of Engineering, Hyderabad, Telangana, India \\ * Corresponding author's Email: solaisbu.phd@gmail.com
}

\begin{abstract}
Nowadays, cloud server adoption becomes more popular and highly demanded due to unlimited data contribution and retrieval from anywhere and anytime. Existing methods have lacks of adaptability issues with a trust value calculation based on multi-dimensional cloud service providers. Some, existing methods believed in expert opinion to evaluate the trust factors. However, the techniques have many adaptability issues and trust evaluation results have many errors. To bring the better solution, Trustworthy and Scalable Service Providers Algorithm is proposed for analyzing the design the relationship among the users, the broker, and cloud service providers. The proposed method works for resource allocation based on matchmaking service among multiple clouds. The trust-based proposed method efficiently minimizes the cloud user burden and enhances the system stabilities. Proposed method works based on information entropy theory to evaluate the multi-attribute based decision-making. Here, cloud user efficiently can find trustable cloud service providers in advance. Where, cloud service providers are more dependent on cloud users. Based on the excremental results, the proposed method reduces the System execution time 2 milliseconds; communication cost $9.33 \%$ and improves the $39.33 \%$ Trust score compare than existing methodologies in multiple cloud environments.
\end{abstract}

Keywords: Trustworthy, Matchmaking services, Multiple cloud environments, Cloud service providers, Cloud users, Trustworthy and scalable service providers algorithm.

\section{Introduction}

Nowadays, the cloud server is become a more demandable and recent trend for industries and enterprises business due to unlimited data storage and accessibility features from anywhere at any time. Cloud users are willing to contribute their most credential and important information in cloud storages. Where, they are looking to establish a trustable relationship between cloud services providers, cloud user, and brokers. The scope of cloud computing has been enhanced with emerging cloud brokers improvement. Where, they are acting as a mediator between cloud users and cloud service providers for negotiating the resource prices and resource allocation among multiple clouds. There are some innovative platforms designed for cloud brokers. The existing methods have a lack of trust and service reliabilities issues between cloud users and cloud service providers. It will seriously hinder the universal acceptance of clouds as outsourced computing services. There are some studies done and focused on a trust-aware brokering framework in multi cloud environments. Where, cloud brokers can work as mediator and aggregation capacities for enabling the cloud service providers to fulfill the requirement across multiple clouds.

Current methods have lack adaptability with a trust fusion evaluation based on multi-dimensional cloud service providers. Where, the weight allocations of multiple cloud service providers adaptively are significant in trust fusion calculation for escaping the effect of individual preferences on weight allocation. There are some existing methods worked based on expert opinion to weight trust factors. However, this approach has lacked 
adaptability issues and it may lead with lot of error in trust evaluation results. The traditional trust algorithm calculates the weight of trusted attributes manually. The rest service and last audit (FSLA) method worked on trust initializations issues for newly registered cloud service providers details and resources. Once, cloud resources register for business, no other cloud user can view previous service history. Here, old cloud service providers are non-existent.

Minimum Critical Path (MCP) method schedules directed acyclic graphs with communication costs in the limited number of resources service providers. Generally, the method used to reduce the complexity of task queue, task execution time. However, the method is cost expensive and not applicable to offer a large amount of cloud resource providers. It also has high communication cost for resource allocation compare than other algorithms. Greedy-Cost algorithm is applicable for a dynamic heterogeneous resource in a cloud environment, which optimizes the scheduling for job execution and resource allotment. The method makes an optimal local preference in resource selection in the cloud. However, Greedycost is time-consuming techniques to execute the cloud user task. Trust Service Oriented Workflow Scheduling (TWFS) algorithm addresses to reduce the execution time in scheduling without consideration of monetary cost and user required resource allocation with reliable effort. TWFS discusses the trust policies and strategies to avoid the obstacles with hybrid workflow scheduling. However, TWFS method can be only used practically for cloud user task execution and well cloud service provider selections. Trust Based Meta Heuristic Workflow Scheduling (TMWS) technique worked to confirm the allocated resources based on execution time, communication cost with the trustworthiness of cloud service providers. Here, the system-integrated mechanism evaluates the trustworthiness of cloud service providers based on context role, flexible collaboration, and their involvement types. However, the method is unable to fulfill the cloud user level trust satisfaction and resource allocation accuracy.

Trustworthy and Scalable Service Providers Algorithm introduces for analyzing the structure design of relationship among the users, the broker, and cloud service providers. The proposed method works for resource allocation based on matchmaking service among multiple clouds. The trust-based proposed method efficiently minimizes the cloud user burden and enhances the system stability. The proposed algorithm evaluates the trustworthiness of cloud service provider based on multi-attribute decision-process. The proposed method evaluates the trustworthiness of cloud services providers and resources based on unilateral trust scale. The proposed algorithm emerges with multiple scales into a trust vector to offer an effective trustworthiness of cloud resources. The proposed method is more reliable with the essential attributes and their trustworthy relationship. Hence, the method is capable of fulfilling current expectations of cloud service providers. The paper contribution is given below as:

$>\quad$ To develop Trustworthy and Scalable Service Providers Algorithm introduces for analyzing the structure design of relationship among the users, the broker, and cloud service providers.

$>\quad$ To evaluate the trustworthiness of cloud service provider based on multi-attribute decision-process

$>\quad$ To offer resource allocation based on matchmaking service among multiple clouds and method emerges with multiple scales into a trust vector to offer effective trustworthiness of cloud resources.

$>\quad$ To improves the cloud service providers' trust score, and reduce the system execution time and communication cost compare than existing methodologies.

The rest of paper is organized as: Section 2 reviews and studies the closest method for trustworthy resource provider services in the cloud. Section 3 describes the proposed methodology, diagrammatic representations, algorithm features, and implementation process. Section 4 discusses deployment setup, dataset, evaluation parameters, and comparative results. Section 5 concludes overall work with the future outcome.

\section{Literature review}

All pace allowed.

The multi-cloud in cloud computing is the combination of two or more cloud data centers that attempt to minimize the data loss or downtime failure in localized cloud systems. The emergence of multi-cloud concept was due to an explosion in the demand of scalable computing. But, the system faces major challenges like privacy, trust, secure data processing of the user etc.

In [1] developed a T-broker scheme (Trustaware service brokering scheme) for matching cloud-based resources or services that satisfy several 
user requests. The T-broker scheme addressed the reduction of networking risk and system efficiency improvement in cloud computing. Nevertheless, the method does not explore anything about trustworthy service recommendations. In [2] intended a systematic trust management scheme in the computation of multi-cloud environments that supported by multi-dimensional resource service operators. The SOTS (service operator-aware trust scheme) evaluated the trust in the cloud resources against the conventional trust schemes that had focused on unilateral trust aspects in cloud resource services. But, the technique does not cover on task execution and efficient resource allocation process. In [3] constructed a Cloud Bursting Brokerage and Aggregation (CBBA) algorithm in clouds for bursting and aggregation process. The method also utilized a secure sharing mechanism technique for sharing the cloud resources on various cloud environments. In [4] constructed a Data-driven and Feedback-Enhanced Trust (DFET) computing technique that achieved multiple data centers mechanism processes. It has highly focused on trustbased computing requirement in multi-cloud collaborative resource services. It also expressed trust-aware monitoring service architecture for serving middleware in multi-cloud trust computing with task scheduling capability, which was based on a distributed soft agent's concept. In [5] constructed a fuzzy system called fuzzy logic based trust evaluation system that accepted the user responses in the form of a fuzzy linguistic system. In the constructed model, it incorporated the fuzzy goal and constraint techniques that predicted trust value following the weight of feedback. However, these methods only concentrate on trust evaluation and trust score of service providers. The method does not discuss about effective resource allocation, job executions which is challengeable tasks in cloud computing.

In [6] expressed a broker oriented cloud service model that discovered services based on consumer requirements. The focus of the study was on service discovery and composition of systems and it discussed the cloud service model included with cloud broker with desirable features. In [7] addressed a multi-factor cloud service credibility evaluation model that evaluated the service credibility of the cloud based on factors like trust evidence, and time. In [8] built a cloud broker (CB) model that helped consumers in selection of appropriate Cloud Provider (CP) that processed the request towards the trust-based environment. The work-utilized feedback, which considered a good source that, evaluated the $\mathrm{CP}$ trust and trustworthiness in the feedback. In [9] constructed a Trust-CAP model (Trust model for cloud-based applications) that addressed trust in cloud computing. It considered four components like integrity, availability, access control, and privacy. The trustcap ensured the achievement of the security services towards several attacks like Man-At-The-End (MATE) attacks. In [10] expressed two models that identified fake feedbacks of users. Initially, it addressed the feedback evaluation model for evaluating the incoming feedbacks and identifying fake that occurs. Hence, it discussed Bayesian game model for detection and preventions of malicious users. But, these methods only discuss about broker activities. However, there is no more work to identify the services provider stabilities and reliabilities in multi-cloud environments.

In [11] expressed a subjective logic trust model based to combine the factors like direct experiences, satisfied interaction feedback, reputation values, and risk factor. The method addressed the problem of creating trust in the cloud services and its evaluation. The method tends to perform comprehensive prioritization on service providers that led the users to compare and choose proper service providers based on their needs. In [12] studied the characteristics of trust like integrity, availability, reliability, dependability, security, dynamicity, scalability, safety, and confidentiality. The work also discussed trust applications like monitoring and tracking. In [13] constructed a distributed and modular perceivable architecture for addressing service behavior of large-scale virtual machines based on the distributed monitoring agents. It further illustrated a parallel trust-computing scheme for big monitoring data. In [14] addressed scheduling schemes in multi-cloud for multi-media services and trust model that included subjective and objective trust that evaluated the degree of trust. It entrusted with multimedia service providers. It employed Bayesian theory and obtained subjective trust degree among multimedia service providers and users. In [15] formulated a grid based resource allocation decision model with service reliability assessment and virtual payment assessment. The work addressed on optimal factor, service expense and service reliability issues by expressing bi-objective soft-computing techniques with PC-GA (Genetic Algorithm) and PC-PSO (Particle Swarm Optimization) in which Pareto-set cluster (PC) and reborn techniques. Here, all above-mentioned techniques about cloud user requirement adoptions and trustworthiness of broker services and behavior of services providers. However, there is no more 


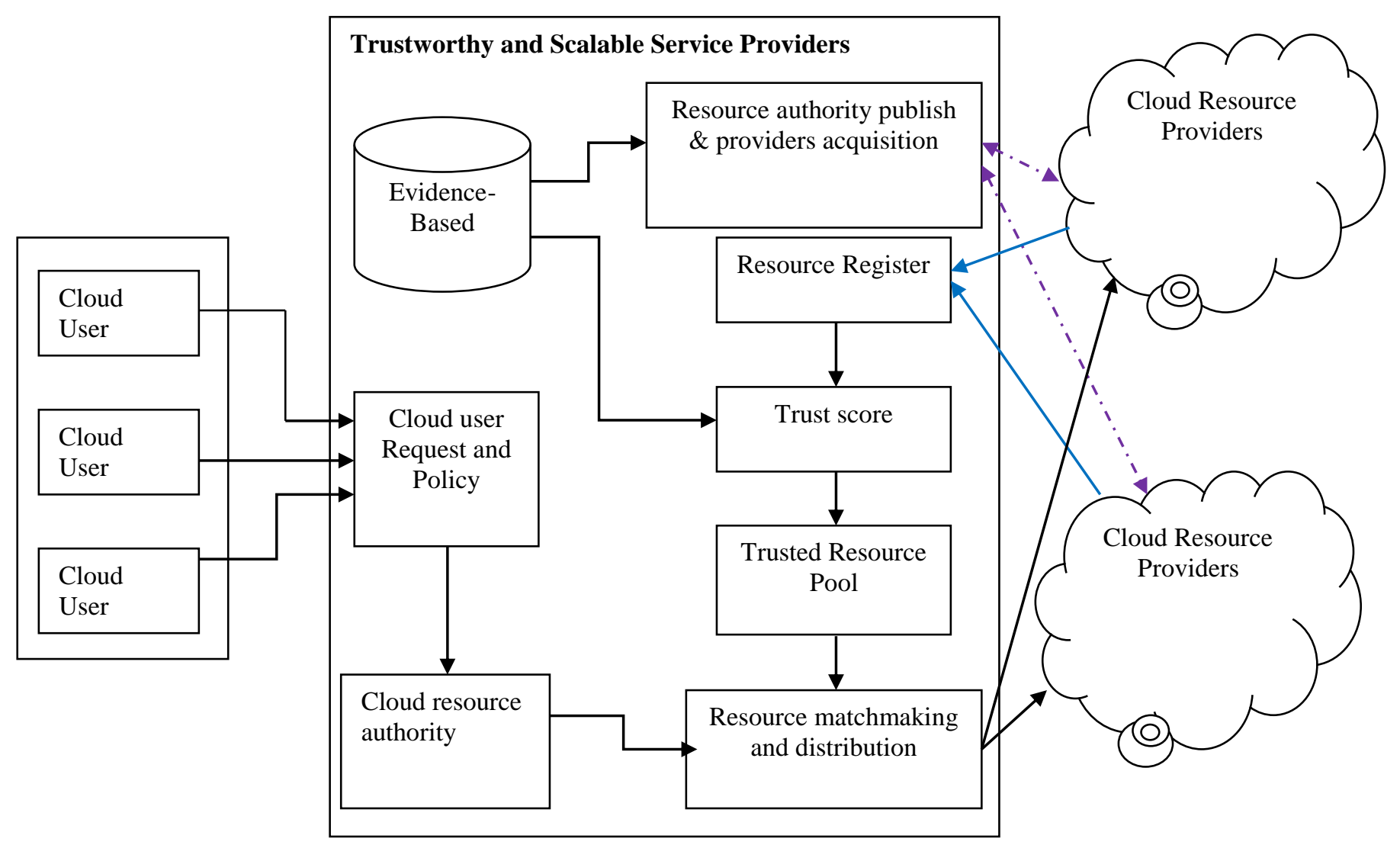

Figure. 1 Workflow representations of trustworthy and scalable service providers algorithm

concentration on efficient resource distributions with trustworthy and reliable cloud service provider, which should be, relied on task executions and communication cost.

In [16] discussed a Democratic Instance Selection (DIS) algorithm for parallel implementation. DIS algorithm achieved less computational complexity, linearity in the number of instances and intuitively parallelized internal configuration. The DIS algorithm attempts to follow the MapReduce model and provided the implementation with the help of popular big data framework called spark. In [17] designed a brokerage-based architecture; where, the cloud brokers are responsible for the service selection. The work also designed B-Cloud Tree an efficient indexing structure that managed the more significant information of cloud service providers. In [18] expressed a cloud service-mapping framework based on two-way ranking. Further, the work evaluated the ranking score of both (Cloud Service Providers) CSPs and (Service Requesting Customers) SRCs in two-way based cloud service mapping framework by utilizing Analytic Hierarchy Process (AHP) with the support of Quality of Service (QoS) parameters. In [19] explained Intelligent Broker (IB) system for service discovery among multiple cloud service providers. The service requirements in the system defined in the precise form of numerical values. The work also built service discovery and Service recommendation algorithm. In [20] described a Trust Management Middleware (TMM) framework with the integration of subjective assessment and objective assessment from users and service monitors respectively for identifying trustworthy in the service selection in the cloud. The credibility of user feedback also determined with the development of covariance based algorithm. In [21] explained the TMWS (Trust based Modeling Workflow scheduling) using as a directed acyclic graph to store the resource information and cloud service providers details. The method worked to confirm the resources based on the time, cost and trust cloud service providers which evaluates based on their previous service histories services to cloud users. The above methods discuss about service providers workflow scheduling and task executions. But, there is very few works are available for reliable and trustworthy cloud service providers selections which is highly committed on task completion time and communications cost in multiple cloud environments. 


\section{Proposed methodology}

Trustworthy and Scalable Service Providers Algorithm proposes to analyze the relationship among the cloud users, brokers, and cloud service providers. The proposed method works for resource allocation depended on matchmaking service among multiple clouds. The proposed method explains system architecture that contains deployment preprocessing steps, including the trusted resource matchmaking services and algorithms feature with pseudo code explanations. Fig. 1 expresses the workflow representations of Trustworthy and Scalable Service Providers Algorithm below in details.

\subsection{Cloud user}

The cloud user can register with their general information and obtain cloud resources for getting trustworthiness of resources and cloud service providers in a cloud environment. The cloud user information is authenticating then access the information. The cloud user demands for the services to the broker with the access policies, consequently the customer requires the particular services for production usability, trustworthiness and protection to the customers.

\subsection{Cloud service providers}

Cloud service provider works a middle person between cloud user and storage server to develop efficient request-response model in a cloud infrastructure. The CSP stores various types of information in a distributed mode on various servers, which physically present in various places. Cloud service providers manage with enterprise environment, and it provides scalable, security and reliable service for cloud users with minimum cost. The cloud service provider gives the different services through the broker so that the broker with trustworthy for the service administrator and to the customer.

\subsection{Cloud resource authority}

With the establishment of trustworthiness and reliabilities of resources, cloud service providers and cloud user authority in cloud environment, cloud resource authority may simultaneously deal with various authority delegations upon various cloud users demands. Cloud resource authority is performing the numerous evaluating tasks concurrently. It manages the available resources, cloud services providers, and cloud request parallel.
It also greatly decreases the computation cost and task allocation scheduling in the cloud environment.

\subsection{Reliable resource allocation}

Reliable Resource Allocation manages and indexes every one of the assets accessible from numerous cloud suppliers, and acquires task from every specific cloud asset, acting as estimating interface for cloud users, and altering the task, when new task request is accessible.

\subsection{Trustworthy and scalable service providers (TSSP) algorithm}

Trustworthy and Scalable Service Providers (TSSP) algorithm introduces for analyzing the trustable and reliable relationship among cloud users, broker, and cloud service providers. The TSSP algorithm works for resource allocation depended on matchmaking service among numerous clouds. The TSSP algorithm estimates the trustworthiness of cloud service provider and resources based on unilateral trust scale. Here, every cloud resource providers registers their service assets through the cloud resource authority. The cloud service user consults with the cloud resource authority on the Service-Level Agreement (SLA) features. The technique ultimately arranges a SLA agreement. Along with the agreement, the broker selects and presents highly trusted or assets to cloud users from the trusted or believed asset pool. The broker can dynamically sort high-performance assets by evaluating the historic asset data as far as giving high-confided assets. Here, cloud resource authority on reviewing the demand and services from the cloud user, coordinates, and broadcasts the task request and mail to the cloud user with requested task details and stored in the cloud environment. The task request transfers to the cloud user with their required services details. Here, Directed Graph $\mathrm{G}=(\mathrm{V}, \mathrm{E})$ prefer to expresses resource distributions and optimizations for task scheduling. V represents the group of virtual machine (VM). Where, while E denotes the set of edges for matchmaking services between cloud and cloud resource service providers. Where, VM have heterogeneous capabilities in terms of VM capacity and total amount of allocated job in queue with trust score. Where, weight is assigned to every service resource providers with outgoing edge from service resource provider A to service resource provider B; where, service resource provider $\mathrm{B}$ is compared with service resource provider A. When, task request receives at datacenter by cloud users. Hence, the proposed method starts to match the cloud user requirement 
with available service resource provider. Where, the method gives first priority those resource providers whose weights are high. Hence, task completion time initiates from VM and all service provider weights are re-evaluated. If VM fails to initiates the task then their weight will be considered as zero. If any allocated task in queue fails to execute then task will be migrated to another VM. The proposed method highly concentrates service reliabilities and trustworthiness of service resource providers during graph connectivity. The cloud resource authority is serving the demand or responding the demand, the service status updates of service providers. Whether, it is processed or it is pending. The cloud resource authority knows about the assets seeking and offering with the matchmaking structure. The method integrates the reputation and feedback of cloud users to concern about trust score.

Reputation is a trust about an entity based on other entities evaluation at specific time and context. Where, Feedback is estimated based on the satisfaction cloud user that can rate the services which are already offer by cloud service providers. The cloud resource authority marks the count of positive and negative feedback based on the cloud user responses. Here, Task Failure rate estimated based on total server capabilities during requestresponses process. For example, cloud service capacities $n=100$, then cloud resource providers have some threshold to handle the cloud user request. The threshold scale $=70$ and Total task handling capacity $=100$; while, processing the new task with cloud resource servers verifies the specified threshold level. Once, threshold level reached the maximum lime then server will decline the new task. The task failure is evaluated to calculate the trust score. Task queue is estimated based on the service utilization. Initially, server is ideal and considered as Server $\mathrm{S} 1=0, \mathrm{~S} 2-=0, \mathrm{~S} 3=0, \mathrm{~S} 4=0$, Where, task request will be transferred to Server S1 based FCFS(First Come First Service). Once, client sends task request then the method will optimize the task load of servers who have least task to complete based time estimation of specific services. If, the allocated server has more task load then task will be transferred to other services that have resource reliability and trust score. Service provider's delay responses are also affects their trust score. The proposed algorithm PA is expressed as P:

$$
\mathrm{P}=\left\{\mathrm{U}_{\mathrm{C}}, \mathrm{R}_{\mathrm{P}}, \mathrm{F}_{\mathrm{S}} \mathrm{R}_{\mathrm{S}}, \mathrm{W}_{\mathrm{S}}, \mathrm{T}_{\mathrm{S}}\right\}
$$

Lets $U_{C}$ denoted as group of registered cloud users, who is looking service in cloud to deploy their applications. It expressed as:

$$
U_{C}=\left\{\mathrm{U}_{\mathrm{C} 1}, \mathrm{U}_{\mathrm{C} 2}, \mathrm{U}_{\mathrm{C} 3} \ldots . . \mathrm{U}_{\mathrm{Cn}}\right\}
$$

Where, $U_{C n}$ is $-n^{\text {th }}$ users of system.

Hence, $R_{P}$ is considered as a cloud service resource provider, which is denoted as $R_{P}$ and it uses for offering the service in cloud environments. Cloud service resource provider is expressed as:

$$
R_{P}=\left\{\mathrm{R}_{\mathrm{P} 1} \mathrm{R}_{\mathrm{P} 2} \mathrm{R}_{\mathrm{P} 3} \cdots \cdots \cdots \cdots \cdot \mathrm{R}_{\mathrm{Pn}}\right\}(3)
$$

Where, $R_{P n}$ is $-\mathrm{n}^{\text {th }}$ mummer service resource provider is cloud environments.

Here, many cloud users are looking for resource service to execute their applications. Cloud user requirement details are shared among available service providers. Simultaneously, available services resource provider details are also shared with cloud user for service selections. Where, cloud user can give their feedback based on the evaluation of various parameters in terms of service reliabilities, trustworthiness, job executions, and communication cost constraints. Here, the proposed technique considers Feedback score $F_{S}$ to respond their opinion behalf of services resource providers. Where, $\mathrm{R}_{\mathrm{S}}$ denotes the reliabilities score of services providers based on the evaluation of multidimensional emulations parameters, task executions, and services scalabilities.

$$
\begin{aligned}
& F_{S}=\left\{\mathrm{F}_{\mathrm{S} 1}, \mathrm{~F}_{\mathrm{S} 2}, \mathrm{~F}_{\mathrm{S} 3} \ldots \mathrm{F}_{\mathrm{Sn}}\right\} \\
& R_{S}=\left\{\mathrm{R}_{\mathrm{S} 1}, \mathrm{R}_{\mathrm{S} 2}, \mathrm{R}_{\mathrm{S} 3} \ldots \mathrm{R}_{\mathrm{Sn}}\right\}
\end{aligned}
$$

Where $F_{S n}$ is feedback score and $R_{S n}$ is reliability score of service resource providers.

Hence $\mathrm{W}_{\mathrm{s}}$ is indicates the aver acre weighted score of the services resources providers which is evaluated based on multidimensional evaluation parameters. Where, each service resource providers have specific weight, which is defined as:

$$
W_{S}=\left\{\mathrm{W}_{\mathrm{S} 1}, \mathrm{~W}_{\mathrm{S} 2}, \mathrm{~W}_{\mathrm{S} 3}, \ldots . \mathrm{W}_{\mathrm{Sn}}\right\}
$$

Where, $W_{S n}$ is weighted score of service resource providers.

Next, Trust Score $\left(T_{\mathrm{S}}\right)$ is calculated based on the average score of reliabilities, weight, scalabilities, task failure and feedback score. Trust is not only 
meaning belief here. However, it also evaluate that how the specific services resource providers are capable to execute the job successfully within give time frame. Trust score is expressed as:

$$
T_{S}=\left\{\mathrm{T}_{\mathrm{S} 1}, \mathrm{~T}_{\mathrm{S} 2}, \mathrm{~T}_{\mathrm{S} 3}, \ldots \mathrm{T}_{\mathrm{Sn}}\right\}
$$

Where, $T_{S n}$ is Trust score of service of services resource providers.

Finally, Trust Score $\left(T_{s}\right)$ denotes the overall trust score of services resource providers based on the evaluations of multi-dimensional parameters for trustworthy and reliable services to cloud users. The overall trust score is evaluated as:

$$
T_{S}=\frac{\left(\sum T_{S 1}+T_{s 2}+T_{s 3} \ldots \ldots \ldots . . T_{S n}\right)}{(n)}
$$

The method integrates the reputation and feedback of cloud users to concern about trust score. The proposed algorithm pseudo code is explained below in details:

Input: Cloud resource provider (CRP), cloud user feedbacks (CUF), size of task (T);

Output: Trust score (TS), System execution time (SET), Communication cost (CC);

Begin

Let Ns be total amount of server;

Calculate servers total score form feedback and save it in total_feedback

Calculate cloud service provider score based on total task queue;

Evaluate task failure score based on total task_failure;

Initialize Trust Score $=0$;

For $\mathrm{i}=1$ to $\mathrm{N}$;

Retrieve feedback score, task queue score \& task failure;

Evaluate Weight_Feedback=Feedba

ck(i)/Total_Feedback

Calculate Weight_TaskQueue $=1$ -

( TaskQueue (i)/Total_TaskQueue)

Calculate Weight_TaskFailure $=1$ -

(TaskFailure(i)/Total_TaskFailure)

Trust

Score=Weight_Feedback+Weight_Queuelo ad+ Weight_TaskFailure

End for

Predict Trust score (TS), System execution time (SET), Communication cost (CC);

End
Table 2. Input parameter details

\begin{tabular}{|c|c|}
\hline Parameters & Value \\
\hline Task Length & $5,000-1,00000$ \\
\hline Number of Task & $5,10, \& 15$ \\
\hline Task File Size & $500-5000$ \\
\hline VM & $512-1024$ \\
\hline Total VM & 10 \\
\hline Total PE per VM & $1-3$ \\
\hline VM Processor & Xen \\
\hline Storage & $1,00000-10,00000$ \\
\hline Virtual Machine Unit Cost & $1-10 \$$ \\
\hline VM Memory & $512-2048$ \\
\hline Bandwidth & $500-1200 \mathrm{MBPS}$ \\
\hline
\end{tabular}

\section{Result and discussion}

\subsection{Deployment setup}

The experiment deployed on a Desktop with Intel $\mathrm{i}^{6}$ Core Processor, 8 GB RAM, 500 GB memory and Window 7 professional operating system. The proposed algorithm is developed on NetBeans 8.0. IDE, 1.8 JDK, Apache Tomcat 8.0.15 with MYSQL 5.5 Database in Java programming languages. The proposed algorithm executed under is Jelastic Cloud Server. The proposed method is evaluated with various types of cloud resource providers and resource allocations. It characterizes the details of 5, 10, and 15 tasks of resources and service provider which is given in Table 1 for the methodology evaluation process.

\subsection{Simulation result}

Here, proposed TSSPA algorithm represent experimental results in tabular and graphical view, which improves the trustworthiness and scalability of cloud services provider's resources and services accuracy in cloud server storage. The proposed algorithm works to evaluate the trustworthiness and reliability of cloud services providers and their resources in a cloud environment on various parameters. This model estimates the system execution time (SET) in milliseconds, communication cost (CC) in percentage $(\%)$ and Trust Score in percentage (\%) with conventional methodologies.

\subsubsection{System execution time (SET)}

The proposed method explains the mathematical expression for task computation time in Eq. (1). The task completion time considers the total time utilization from task request receive from cloud user to until respond their requested task time duration. 
Table 1. System Execution Time (SET) in milliseconds (msec), Communication Cost (CC) in Percentage (\%) and Trust Score (TS) in Percentages (\%) for 5 Tasks, 10 Tasks and 15 Tasks

\begin{tabular}{|l|l|l|l|l|l|l|l|l|l|l|}
\hline \multirow{2}{*}{ Techniques } & \multicolumn{3}{|l}{ 5 Tasks } & \multicolumn{1}{|c|}{ 10 Tasks } & \multicolumn{2}{|c|}{ 15 Tasks } \\
\cline { 2 - 12 } & SET & CC & TS & SET & CC & TS & SET & CC & TS \\
\hline Greedy-cost & 3 & 9 & X & 12 & 43 & X & 12 & 58 & X \\
\hline MCP & 1 & 10.5 & $X$ & 8 & 47 & $X$ & 9 & 62 & X \\
\hline TWFS & 3 & 10 & 53 & 10.5 & 46 & 56 & 11 & 62 & 57 \\
\hline TMWS & 2 & 9.5 & 50 & 10 & 44 & 48 & 10 & 59 & 48 \\
\hline TSSPA & 0.5 & 7 & 90 & 6 & 30 & 96 & 8 & 45 & 98 \\
\hline
\end{tabular}

Where, many processes exist like a task receiving, job allocation, resource allocation, job queue scheduling, task processing, and task reply to concern users. In this step, proposed techniques evaluate as system execution times behalf of resource allocation, and job completions in the cloud. Here, system execution time (SET) represents as:

$$
S E T=T_{A R} \times T_{A C T}
$$

Where, $T_{A R}$ is a total number of available task and $T_{A C T}$ is average computation time for the available record.

\subsubsection{Communication cost}

The communication cost is the evaluation of total task request and response services communications in a cloud environment. Communication cost relies the resource utilization, cloud datacenter processing, networking cost, storage cost, bandwidth cost, and overall data transformation from cloud resource provider to cloud users. Where, communication is calculated based on the average value of task completion time and data size. The proposed TSSP approach describes a mathematical model in Eq. (10) to communication cost in percentage (\%). The communication cost (CC) is evaluated as respected of task completion with task sizes

$$
C C=\frac{R_{\text {taskcomp }}}{\text { TCon }_{\text {size }}} \times 100
$$

Where, $R_{\text {taskcomp }}$ is data task completion, and $T_{\text {Consize }}$ is the total size of the task.

\subsubsection{Trust score (TS)}

Trust score is estimated based on certain parameters of cloud service providers and resource allocation accuracy based on reputation, reliability, security, scalability, dependability, competence etc. Here, cloud user feedback evaluations, task failure, and on task queue in cloud server are play vital role to evaluate the trust score. The trust is calculated based on the average evaluation of the reliability, scalability, feedback and task efficiency of cloud service providers and their allocated resource accuracy for cloud user request in (3). The trust score is expressed as:

$$
\begin{aligned}
& T R_{\text {Score }}=W_{\text {Feedback }}+W_{\text {Taskqueue }}+W_{\text {Failure }} \\
& W_{\text {Feedback }}=\frac{1-\text { Feeback }(i)}{\text { Total_Feedback }} \\
& W_{\text {Taskqueeue }}=\frac{1-\text { Taskqueue }(i)}{\text { Total_Taskqueue }} \\
& W_{\text {Failuure }}=\frac{1-\text { Failuretask }(i)}{\text { Total_Failuretask }}
\end{aligned}
$$

Table 2 explains the System Execution Time (SET) in milliseconds (msec), Communication Cost (CC) in Percentage (\%) and Trust Score (TS) in Percentages (\%) for 5 Tasks, 10 Tasks and 15 Tasks. Here, the proposed Trustworthy and Scalable Service Providers Algorithm (TSSPA) is evaluated with following conventional methods namely trust based Meta heuristic workflow scheduling (TMWS)[21], Trust Service Oriented Workflow Scheduling (TWFS)[21], Minimum Critical Path (MCP)[21] Greedy-Cost[21]. Where, Proposed TSSPA is competed by MCP[21] behalf of SET, Greedy-Cost[21] behalf of CC and TWFS[21] in terms of TS for overall tasks. However, their predicted performance is so far. The conventional technique worked on only broker responsibilities, resource allocation, and task execution in cloud 


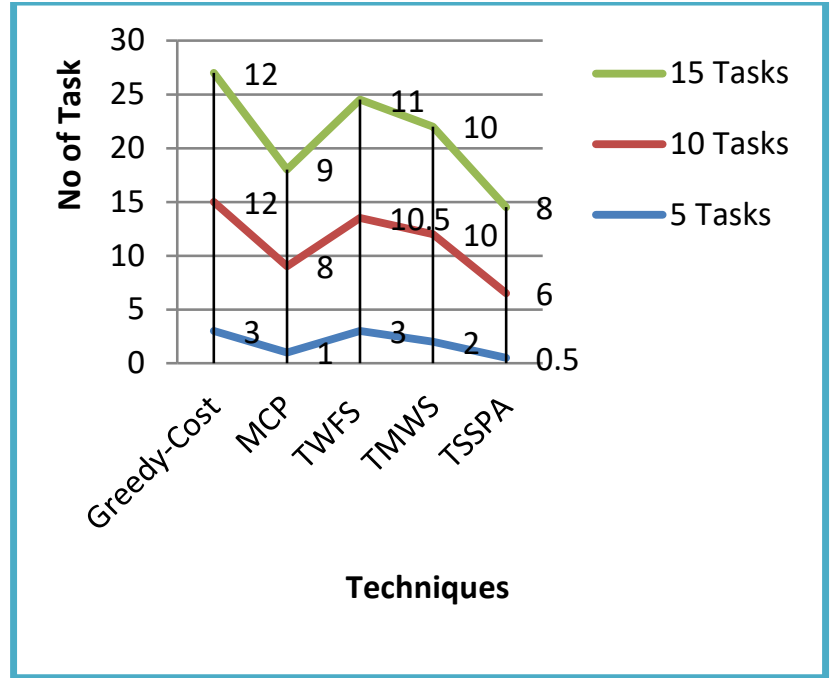

Figure. 2 System Execution Time (SET) in milliseconds (msec) for 5 Tasks, 10 Tasks and 15 Tasks

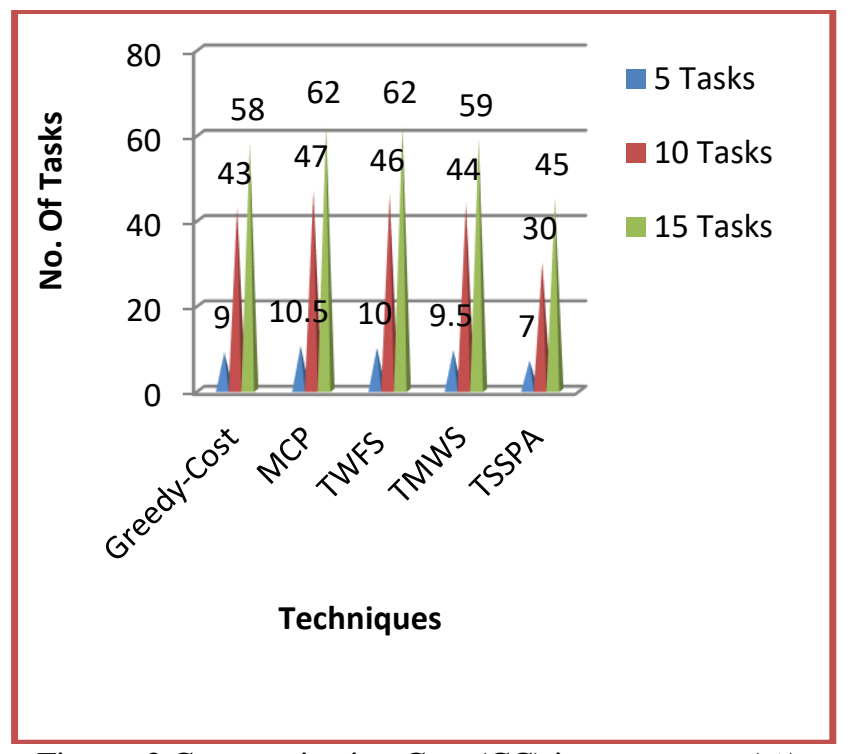

Figure. 3 Communication Cost (CC) in percentage (\%) for 5 Tasks, 10 Tasks and 15 Tasks

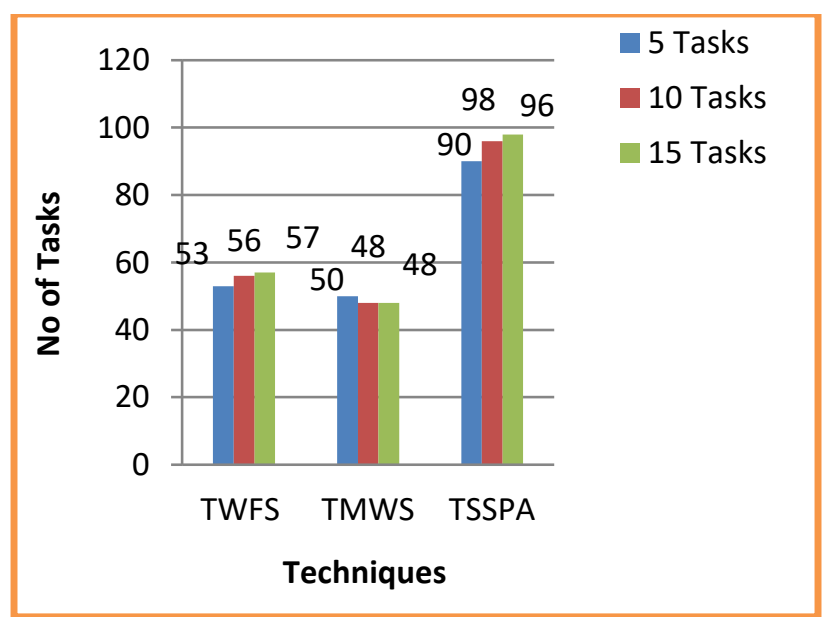

Figure. 4 Trust score (TS) in percentage (\%) for 5 Tasks, 10 Tasks and 15 Tasks environments. There are no works available to offer reliable and trustworthiness service provider's recommendations with effective task execution, load, and resource optimization with minimal communication and time constraints. The method used matchmaking services to find exact resource matching for registered cloud user requirements. Hence, it can be stated that Proposed TSSPA is best on respective parameters to compare than existing methods.

According to Figs. 2 to 4, proposed Trustworthy and Scalable Service Providers Algorithm (TSSPA) is compared on SET, CC and TS for 5,10,15 tasks in the cloud. Proposed TSSP Algorithm is evaluated with TMWS [21], TWFS [21], MCP [21], and Greedy-Cost [21]. In terms of system execution time (SET), a proposed TSSP algorithm is competed by MCP [21]. Where, MCP [21] allocates Directed Acyclic Graphs with communication costs in the limited volume of resources service providers. Generally, the method used to minimize the complexity of task queue, task execution time. However, the method is cost expensive and not applicable to offer a large amount of cloud resource providers. The communication cost is competed by Greedy-Cost [21]. Greedy-Cost [21] algorithm is applicable for a dynamic heterogeneous resource in a cloud environment which optimizes the scheduling for job execution and resource allotment. The method makes an optimal local preference in resource selection in the cloud. However, Greedycost [21] is time-consuming techniques to execute that the cloud user task. Behalf of trust score proposed TSSP algorithm is closely competed by TWFS [21]. TWFS [21] explained to minimize the completion time in scheduling without consideration of monetary cost and user required resource allocation with reliable effort. Where, TWFS [21] elaborates the trust policies and strategies to avoid the obstacles with hybrid workflow scheduling. However, TWFS [21] can be utilized practically for cloud user task execution and well cloud service provider selections. TMWS [21] addressed to assure the allocated resources based on execution time, communication cost with the trustworthiness of cloud service providers. Here, the system-integrated mechanism evaluates the trustworthiness of cloud service providers based on context role, flexible collaboration, and their involvement types. However, the method is unable to fulfill the cloud user level trust satisfaction and resource allocation accuracy.

The proposed TSSP method works for reliable and trustworthy service provider recommendations and resource allocation based using matchmaking service in multi-cloud environments. Where, the 
algorithm concentrates to find exact resource to cloud user based on their service requirements. The method also considers the efficient task executions, load optimization and proper resource utilization to execute task with minimal cost and time. The Here, task completion time considers to evaluate that how proposed method effectively execute the task and respond the cloud user service in minimal time and cost. Proposed method considers request-response interaction and data enter processing time as a task completion time. Communication cost is very important aspects in cloud for user to deploy their application. Proposed method highly concentrates on resource optimization and minimization to reduce the cost. The proposed algorithm also works to reduce task failures, and task queue waiting time.

Proposed method calculates the trust score of service resource provider based on evaluation of the feedback score, reliability score, weighted score and task failures. The proposed always attempts to offers reliable and trustworthy service provider's details based user requirement matching. The technique calculates trust and reputation of the resource providers based on resource availabilities and previous work performances details. Trust-based proposed method efficiently minimizes the cloud user burden and enhances the system stability. The proposed algorithm evaluates the trustworthiness of cloud service provider based on multi-attribute decision-process. The trust-based proposed method efficiently minimizes the cloud user burden and enhances the system stability. Proposed method reduces the System execution time 2 milliseconds; communication cost $9.33 \%$ and improves the $39.33 \%$ Trust score compare than other existing methods. Finally, it can be said that the Proposed TSSP Algorithm is best on respective parameters and task compare than existing methods.

\section{Conclusion}

The article presents Trustworthy and Scalable Service Providers Algorithm for analyzing the design the relationship among the users, the broker, and cloud service providers. The proposed method works for resource allocation based on matchmaking service among multiple clouds. The trust-based proposed method efficiently minimizes the cloud user burden and enhances the system stability. The method is capable of maintaining reliability scalability of resources of cloud service providers and allocated resources. The proposed algorithm evaluates the trustworthiness of cloud service provider based on multi-attribute decision-process. Proposed method reduces the System execution time
2 milliseconds; communication cost $9.33 \%$ and improves the $39.33 \%$ Trust score compare than other existing methods. Finally, it can be said that the Proposed TSSP Algorithm is best on respective parameters and task compare than existing methods.

In future, the paper can be extended to apply the trustworthiness for cloud resource provider with public and private key services in a Hadoop environment. Where, scalability and reliabilities are major challenges in Hadoop environment during multiple cloud server utilization.

\section{References}

[1] R. X. Li, H. Ma, F. Zhou, and W. Yao, "Tbroker: A trust-aware service brokering scheme for multiple cloud collaborative services", IEEE Transactions on Information Forensics and Security, Vol.10, No.7, pp. 1402-1415, 2015.

[2] S. Boddupalli and V. Kanchan, "Multi-Cloud Computing-A Framework of Service OperatorAware Trust Scheme for Resource Matchmaking", International Journal of Advanced Technology and Innovative Research, Vol.07, No. 10, pp.1851-1855, 2015.

[3] P. Jain, D. Rane, and C. Patidar, "A novel cloud bursting brokerage and aggregation (CBBA) algorithm for multi cloud environment", In: Proc. of 2012 Second International Conference Advanced Computing \& Communication Technologies, pp. 383-387, 2012.

[4] X. Li, H. Ma, W. Yao, and X. Gui, "Datadriven and feedback-enhanced trust computing pattern for large-scale multi-cloud collaborative services", IEEE Transactions on Services Computing, Vol. 11, No.4, pp. 671-684,2018.

[5] R. Nagarajan, S. Selvamuthukumaran, and R. Thirunavukarasu, "A fuzzy logic based trust evaluation model for the selection of cloud services", In: Proc. 2017 International Conference of Computer Communication and Informatics, pp.1-5, 2017.

[6] N. Rajganesh and T. Ramkumar, "A review on broker based cloud service model", Journal of Computing and Information Technology, Vol.24, No.3, pp.283-292, 2016.

[7] Z. Li, L. Liao, H. Leung, B. Li, and C. Li, "Evaluating the credibility of cloud services", Computers \& Electrical Engineering, Vol.58, pp.161-175, 2017.

[8] P. Varalakshmi, T. Judgi, and D. Balaji, "Trust Management Model Based on Malicious Filtered Feedback in Cloud", In: Proc. of International Conference on Data Science Analytics and Applications, pp. 178-187, 2017. 
[9] E. Abdallah, G. Zulkernine, M.Y.X. Gu, and C. Liem, "TRUST-CAP: A Trust Model for Cloud-Based Applications", In: Proc. of 2017 IEEE 41st Annual Computer Software and Applications Conference, pp. 584-589, 2017.

[10] S. Siadat, A.M.Rahmani, and H. Navid, "Identifying fake feedback in cloud trust management systems using feedback evaluation component and Bayesian game model", Journal of Supercomputing, Vol.73, No.6, pp. 26822704, 2017.

[11] N. Agheli, B. Hosseini, and A. Shojaee, "A trust evaluation model for selecting service provider in cloud environment", In: Proc. of 2014 4th International eConference Computer and Knowledge Engineering, pp. 251-255, 2014.

[12] M. Chiregi and N.J. Navimipour, "A comprehensive study of the trust evaluation mechanisms in the cloud computing", Journal of Service Science Research, Vol. 9, No. 1, pp.1-30, 2017.

[13] X. Li, J. Yuan, H. Ma, and W. Yao, "Fast and Parallel Trust Computing Scheme Based on Big Data Analysis for Collaboration Cloud Service", IEEE Transactions on Information Forensics and Security, Vol.13, No. 8, pp. 1917-1931, 2018.

[14] Y. Liu, C. Li, Y. Luo, Y. Shao, and J. Zhang, "Scheduling multimedia services in cloud computing environment", Enterprise Information Systems, Vol.12, No.2, pp.218235,2018.

[15] S.C. Wei and W.C. Yeh, "Resource allocation decision model for dependable and costeffective grid applications based on Grid Bank", Future Generation Computer Systems, Vol. 77, pp. 12-28, 2017.

[16] A. Arnaiz-González, A. González-Rogel, J.F. Díez-Pastor and C. López-Nozal, "MR-DIS: democratic instance selection for big data by MapReduce", Progress in Artificial Intelligence, Vol. 6, No. 3, pp.211-219, 2017.

[17] D. Lin, A.C. Squicciarini, V.N. Dondapati, and S. Sundareswaran, "Cloud brokerage architecture for efficient cloud service selection", IEEE Transactions on Services Computing, Vol. 12, No. 1, pp. 144 - 157, 2016.

[18] N. Yadav and M.S. Goraya, "Two-way ranking based service mapping in cloud environment", Future Generation Computer Systems, Vol. 81, pp. 53-66, 2018.

[19] R. Nagarajan, R. Thirunavukarasu, and S. Shanmugam, "A cloud broker framework for infrastructure service discovery using semantic network", International Journal of Intelligent Engineering and Systems, Vol. 11, No. 3, pp.11-19, 2018.

[20] M.B. Smithamol and S. Rajeswari, "TMM: Trust Management Middleware for Cloud Service Selection by Prioritization", Journal of Network and Systems Management, Vol. 27, No. 1, pp. 66-92, 2019.

[21] G. J. Rathanam and A. Rajaram, "Trust Based Meta-Heuristics Workflow Scheduling in Cloud Service Environment", Circuits and Systems, Vol. 7, No. 4, pp.520-531, 2016. 